\title{
Le paysage lacustre par le prisme du tourisme : le cas du lac d'Aiguebelette (Alpes, France)
}

The lake landscape through the prism of tourism: the case of Lac d'Aiguebelette (Alps, France)

Laura Rudzewicz, Yoann Baulaz et Véronique Peyrache-Gadeau

\section{OpenEdition}

\section{Journals}

Édition électronique

URL : http://journals.openedition.org/tourisme/2936

DOI : 10.4000/tourisme.2936

ISSN : 2492-7503

\section{Éditeur}

Éditions touristiques européennes

\section{Référence électronique}

Laura Rudzewicz, Yoann Baulaz et Véronique Peyrache-Gadeau, « Le paysage lacustre par le prisme du tourisme : le cas du lac d'Aiguebelette (Alpes, France) », Mondes du Tourisme [En ligne], 17 | 2020, mis en ligne le 01 juin 2020, consulté le 25 décembre 2020. URL : http://journals.openedition.org/ tourisme/2936; DOI : https://doi.org/10.4000/tourisme.2936

Ce document a été généré automatiquement le 25 décembre 2020.

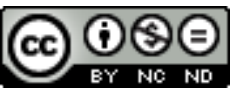

Mondes du tourisme est mis à disposition selon les termes de la licence Creative Commons Attribution - Pas d'Utilisation Commerciale - Pas de Modification 4.0 International. 


\section{Le paysage lacustre par le prisme du tourisme : le cas du lac d'Aiguebelette (Alpes, France)}

The lake landscape through the prism of tourism: the case of Lac d'Aiguebelette (Alps, France)

Laura Rudzewicz, Yoann Baulaz et Véronique Peyrache-Gadeau

Dans les Alpes, les lacs sont devenus des lieux d'attractivité touristique et l'environnement lacustre en a été modifié: le paysage est désormais fortement déterminé par l'influence touristique. Comprendre les interactions entre les pratiques récréatives et les dimensions environnementales spécifiques à l'espace lacustre permet d'éclairer la place du tourisme, jusqu'à reconfigurer le paysage. Paradoxalement l'empreinte touristique et la spécificité des pratiques et des représentations, qui sont maintenant associées aux paysages lacustres, restent assez peu étudiées par les sciences humaines et sociales en dehors du point de vue de la géographie culturelle, qui s'intéresse à l'histoire des liens entre lacs et société. Cet article s'appuie sur les acquis de cette géographie pour aborder l'observation d'un espace désormais placé sous influence touristique: celui du lac d'Aiguebelette, situé en Savoie dans les Alpes françaises (cf. figure 1). 
Figure 1. Le lac d'Aiguebelette dans le réseau des grandes villes françaises des Alpes du Nord

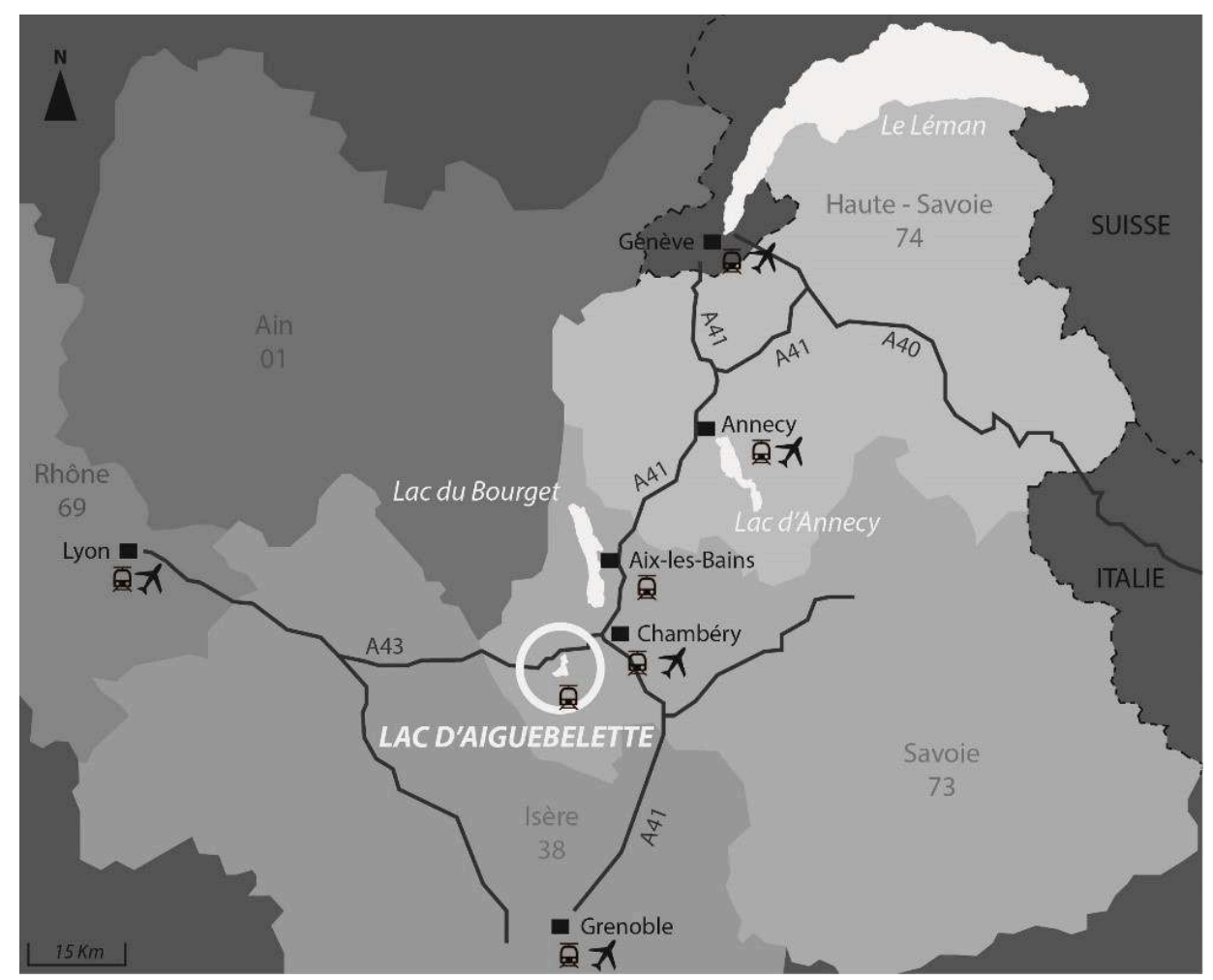

Source : Google Earth, IGN ; réalisation des auteurs, 2018

2 L'objectif théorique est d'envisager la caractérisation du paysage lacustre à partir de sa dimension physique et environnementale, en incluant à la fois les aménagements et usages touristiques et l'expérience sensible des lieux. Quelles composantes paysagères portent la marque, voire sont conditionnées, par des pratiques touristiques? En quoi ces pratiques participent à spécifier le paysage lacustre? Quelles représentations en ont les usagers? Peut-on identifier un cadre cohérent pour répondre à ces questions et définir le paysage lacustre en tant qu'entité environnementale, aménagée, pratiquée et perçue du point de vue du tourisme?

Quatre étapes d'analyse, à partir du cas du lac d'Aiguebelette, sont tour à tour présentées ; elles sont porteuses, chacune, d'une approche du paysage lacustre et d'une méthodologie propre d'observation. Dans une première partie, on propose de caractériser celui-ci à partir des travaux en géographie culturelle qui se sont intéressés aux relations lac/société, notamment par le prisme du tourisme. Dans une deuxième partie, sur la base d'une cartographie, on envisage la place qu'occupe le lac selon différentes aires de vues, tenant compte du couvert végétal et d'un inventaire des espaces touristiques aménagés. Cette cartographie permet d'établir le cadre-support de l'analyse du paysage caractérisé selon un zonage dit d'influence lacustre par le prisme du tourisme. Dans une troisième partie, on conduit l'analyse spatiale des pratiques touristiques. À partir d'observations de terrain, on caractérise le paysage lacustre par des pratiques différenciées en fonction de la proximité au plan d'eau. Cette différenciation est mise en regard du zonage établi en deuxième partie. Enfin, dans une quatrième partie, on mobilise l'approche en termes de géographie émotionnelle à partir d'entretiens réalisés in situ visant à recueillir des récits associés à la pratique des lieux 
par des acteurs et usagers de l'espace du lac. On analyse les expériences sensibles décrites et, à travers elles, les axes de représentations et les enjeux portés sur le paysage lacustre.

4 Finalement, la possibilité d'articuler les dimensions physiques, pratiques et les représentations du paysage lacustre par le prisme du tourisme est discutée.

\section{Le paysage lacustre en géographie culturelle}

5 Le lac d'Aiguebelette connaît une fréquentation qui le place au rang des principaux sites de loisirs estivaux pour les habitants des centres urbains régionaux proches (Rhône et Savoie). Cette attractivité est rendue possible par une accessibilité facilitée par le passage à proximité, dès 1974, de l'axe autoroutier reliant Lyon à Chambéry dans les Alpes du Nord ${ }^{1}$. Ce lac est même devenu ces dernières années une aire d'attraction saisonnière qui conduit à une saturation de ses plages et des parkings les fins de semaines caniculaires. Cette fréquentation du littoral pourrait remettre en cause les nombreuses distinctions patrimoniales associées au lac d'Aiguebelette. Dès les années 1930, le site a fait l'objet d'une inscription à l'inventaire des sites classés, puis, notamment, d'un arrêté préfectoral de protection de biotope (APPB) en 1990, d'une intégration aux zones d'intérêt écologique faunistique et floristique (ZNIEFF) de type I et II en 1992, le site a été intégré au réseau Natura 2000 en 2006 et, plus récemment, en 2015, le lac et une partie de son bassin versant ont été classés en réserve naturelle régionale (RNR). La préservation des milieux naturels permet notamment aux communes proches de profiter d'une eau potable de qualité et peu traitée (Jargot et Loup, 1983 ; Tissut, 2000, 2002). Les dispositifs de protection ont été progressivement mis en place au cours du $\mathrm{xx}^{\mathrm{e}}$ siècle et leur gestion est aujourd'hui prise en charge par les collectivités ${ }^{2}$, en relation notamment avec le Conservatoire des espaces naturels, avec le souci de préserver le contexte de lac naturel dans sa globalité. Cette gestion est conduite dans un cadre intercommunal regroupant dix communes ${ }^{3}$. Les collectivités disent se reconnaître dans ce territoire fondé sur cette composante identitaire qu'est le lac: «La qualité et la richesse environnementale du site constitue le caractère identitaire dominant du territoire et certainement l'un des mieux perçus par le grand public.» (CCLA, 2009, p. 22)

6 Ce contexte questionne l'articulation des dimensions à la fois environnementale et sociétale du paysage lacustre. En limnologie ${ }^{4}$, en géographie physique des lacs (Rougier, 2000), l'identité propre à chaque lac est, en partie, déterminée par son fonctionnement et par les relations entre le bassin versant et le plan d'eau (Touchart, 1993, 2000). De ce point de vue, les lacs sont caractérisés par des dimensions physiques et biologiques, et en tant que systèmes fragiles qui combinent les effets de la domination du bassin hydrographique et l'autonomie relative de leur écosystème spécifique. Cette approche doit cependant être complétée par une autre dimension, qui intéresse la géographie culturelle, qui est celle de la relation au paysage et des représentations que celle-ci suscite. On retiendra dans ce champ les travaux qui s'intéressent: i) aux liens entre sociétés et paysages lacustres; ii) aux lacs en tant que paysages touristiques; et iii) aux travaux de géographie émotionnelle qui portent sur l'expérience sensible du paysage. 


\section{Les liens entre sociétés et paysages lacustres}

7 La géographie culturelle (Cosgrove, 1989 ; Claval, 1992, 2012) aborde les paysages à partir des processus naturels et sociaux qui les construisent. Dans cette perspective, le paysage lacustre est un résultat de l'action humaine, qui s'inscrit de manière visible dans l'environnement. Cette approche du paysage permet une lecture d'un point de vue objectivé pour interpréter la relation des sociétés à leur environnement dans une perspective holistique. Le paysage est décrit par l'articulation de plusieurs plans (du proche au lointain), en prenant appui sur des objets de différentes natures (biotiques, abiotiques et anthropiques), et en tant que support portant les traces d'éléments caractéristiques des dimensions fonctionnelles et symboliques des sociétés (Claval, 1992). Cette approche a notamment été défendue par Augustin Berque, pour qui la géographie culturelle est « l'étude du sens (global et unitaire) qu'une société donne à sa relation à l'espace et à la nature; relation que le paysage exprime concrètement " (Berque, 1984, p. 33) ; et en se fondant sur une explicitation du "paysage-empreinte " et du « paysage-matrice » :

Le paysage est une empreinte, car il exprime une civilisation; mais c'est aussi une matrice, car il participe des schèmes de perception, de conception et d'action c'est-à-dire de la culture - qui canalisent en un certain sens la relation d'une société

à l'espace et à la nature, donc le paysage de son œcoumène. Et ainsi de suite, par d'infinies boucles de codétermination. (Ibidem, p. 33).

Ce souci d'une approche du paysage qui intègre à la fois des connaissances objectivées, projetées sur un espace physique matériel, et des appréhensions subjectives liées à la connaissance intime et pratique des lieux, a été questionné et débattu dans la littérature (Bertrand, 1978; Rougerie et Beroutchachvili, 1991; Luginbühl, 1989; Aronson et Le Floc'h, 1996). Mais, face à la difficulté d'établir une méthode unifiée interdisciplinaire, les études se construisent surtout à partir de différentes méthodes complémentaires, inspirées notamment des travaux de l'École nationale supérieure du paysage de Versailles (Bontron, Luginbühl et Cros, 1991).

$\mathrm{Au}$ sein de cette littérature, les paysages lacustres sont assez peu traités. Ils sont cependant pris en compte dans la typologie des espaces investis par la géographie du tourisme (Lozato-Giotart, 2008). La " riviera lacustre » figure ainsi un « modèle alpin » de développement touristique bien identifié : elle se construit à partir du XVIII ${ }^{e}$ siècle par la villégiature d'une élite aristocratique qui s'installe dans des stations calquées sur les rivieras maritimes, où cohabitent palais, palaces, casinos, et qui contribue à promouvoir la valorisation esthétique des paysages lacustres par les arts - via les peintres et les romanciers. Le Léman, le lac d'Annecy et le lac Majeur sont cités en référence. Sur ces grands lacs alpins, les stations thermales se développent et de nouvelles pratiques balnéaires et nautiques s'affirment. $\mathrm{Au} \mathrm{xx}^{\mathrm{e}}$ siècle, la natation introduit une dimension sportive et un rapport nouveau à l'eau, devenue objet d'expériences ludiques et de bien-être. Avec cette pratique, se développe le concept moderne de plage, lieu pour la relaxation et l'hédonisme progressivement aménagé sur le littoral lacustre. Jean-Pierre Lozato-Giotart qualifie la « riveria lacustre » de modèle spatial touristique particulier, dans lequel « les conditions naturelles pèsent d'un poids capital dans l'installation et le développement des stations touristiques lacustres » et sur lequel le tourisme de masse s'est greffé en générant un paysage commun où les « formes d'extensions spatiales sont souvent linéaires, épousant les rives lacustres, en front de lac ou en retrait, selon les possibilités d'installations initiales » (Lozato-Giotart, 
2008, p. 119-120). Par la suite, au cours du $x^{e}$ siècle, les paysages, notamment ceux des grands lacs alpins, connaissent des changements importants, sous la pression de l'urbanisation et du développement touristique, dont les guides produisent une représentation parfois «stéréotypée d'une pièce d'eau trop bien domestiquée et dont l'usage et l'accès sont limités par l'image » (Lévy, Matos et Raffestin, 1998, p. 86).

Mais tous les lacs ne s'inscrivent pas dans cette trajectoire et Jean-Pierre Lozato-Giotart (2008) identifie un autre modèle, dit «élémentaire » ou " embryonnaire », qui échappe au développement touristique (partiellement ou, au moins, pour un temps) pour des raisons climatiques, liées au défaut d'accessibilité, à l'éloignement des grands foyers attractifs et de peuplement, ou encore parce qu'ils font l'objet de dispositifs de protection. Ces lacs sont investis, de façon souvent saisonnière, par des activités qualifiées aujourd'hui de « tourisme de nature».

\section{L'invention du lac comme paysage touristique}

11 D'autres travaux (Vernex, 1985, 1989, 1993, 1998 ; Bergeron, 1987 ; Bergeron et Buisson, 1988 ; Ferrata, 2007) se sont intéressés à l'invention du paysage littoral à partir des aménagements qui ont porté le développement touristique des lacs. Camping, résidences secondaires et hôtels, routes, parkings, sentiers aménagés, panneaux, embarcadères et plages balisent l'espace touristique lacustre d'équipements physiques qui agissent comme de véritables marqueurs du paysage. L'identité lacustre prend, dans ce cadre paysager construit par l'activité touristique, des traits spécifiques : JeanClaude Vernex évoque une "fonctionnalisation de la nature », le lac étant identifié à un "support d'activités et partenaire sportif » au cours du Xx siècle (Vernex, 1993, p. 21).

En fait, la promotion du paysage lacustre par les premiers guides touristiques s'est fondée au départ sur une toute autre image: celle d'une nature idéalisée, voire sublimée par l'influence des figures romantiques, dans laquelle le lac occupe une place centrale, dans un écrin naturel dominé par la ligne de montagnes, mêlant images champêtres, romantisme des rivages et grandeur des sommets. Elle est, jusqu'au début $\mathrm{du} \mathrm{xx}^{\mathrm{e}}$ siècle, une véritable ressource iconographique des destinations des Alpes. Cette image du «lac contemplatif», identifiée par Jean-Claude Vernex et Michèle Martin (2009) à partir de l'analyse des affiches publicitaires touristiques du lac d'Annecy, évolue ensuite au cours du xxe siècle vers celle du «lac actif ", associée à la diffusion des pratiques sportives nautiques, qui s'ajoute à la notion de « lac pur» liée aux efforts de dépollution entrepris à partir des années 1970. Au début du xxi siècle, c'est le «lac actif » qui est toujours au centre de la promotion, mais avec de nouveau un sens plus contemplatif de « lac naturel », en associant le « lac-patrimoine " à son environnement montagneux (Vernex et Martin, 2009).

Le lac d'Aiguebelette, quant à lui, est resté peu présent dans l'ensemble de l'iconographie touristique des Alpes. Il est identifié comme un lieu en retrait, à l'écart des sites les plus attractifs de Savoie. Les premières citations identifiées dans les guides touristiques lui attribuent néanmoins du charme. Chloé Jobert (2017), à partir de la littérature viatique, indique que l'on trouve dans le Guide Joanne de 1863 la mention suivante: "charmant lac d'Aiguebelette dominé à l'Est par des rochers où s'ouvrent grottes et cascades; et à l'Ouest des prairies et des bois ». Victor-Eugène ArdouinDumazet, dans son ouvrage Voyage en France : Le Rhône du Léman à la mer publié en 1903, présente le lac d'Aiguebelette comme une «nappe solitaire, sans ville sur les bords, à 
peine parcourue par de rares barques de pêcheurs, d'un effet magique "; il ajoute : " peu visité encore, malgré la grâce de ses rivages, la beauté de ses eaux et la fraîcheur de ses campagnes, le lac d'Aiguebelette deviendra sans doute un des lieux de rendezvous des touristes qui se portent de plus en plus vers les sites du beau pays de France " (cité par Jobert, 2017, p. 23). De fait, le lac est devenu, à la fin du $x^{e}$ siècle, l'objet fédérateur du territoire, construit en destination touristique aujourd'hui dénommée «Pays du lac d'Aiguebelette » et, grâce à ses qualités naturelles attractives, il est désormais une ressource de nature géotouristique ${ }^{5}$ (Cayla, 2010; Duval et Gauchon, 2010). Ce positionnement au sein d'une stratégie de valorisation territoriale marque un changement d'approche : le paysage, d'abord centré sur le lac en tant que destination à laquelle s'arriment les activités touristiques, devient un paysage porteur de contenus (écologiques, patrimoniaux) objectivés par des dispositifs de protection, mais aussi terrain d'expériences sensibles dont la compréhension appelle le recours à une autre approche paysagère, celle de la géographie émotionnelle.

\section{L'approche du paysage par la géographie émotionnelle}

L'approche du paysage lacustre par la géographie émotionnelle combine objectivation et perception dans une relation d'étroite interdépendance. La proposition a été amenée par Augustin Berque, pour qui le paysage est une donnée sensible pour le sujet avec lequel il entre en relation et qui mobilise pour cela deux dimensions nécessairement imbriquées: celle des composantes matérielles et visibles, et celle des relations immatérielles et invisibles.

Cette conjonction de la réalité factuelle et de l'attente sensible, c'est la motivation qui fait qu'une certaine société aménage son milieu dans un certain sens, et que les individus appartenant à cette société en perçoivent le sens, le reconnaissent et l'apprécient. (Berque, 1995, p. 33).

15 Les travaux de géographie émotionnelle prennent en considération les relations entre les individus et les espaces vécus, ainsi que le rôle des émotions dans la construction des représentations associées au paysage. Dès lors, ils se confrontent aux analyses exclusivement rationnelles (Jones, 2016). L'approche est centrée sur des observations $\mathrm{du}$ lieu et du paysage à travers la valorisation des «[...] topographies de la vie quotidienne [...] imprégnées d'émotions et de sentiments » (Nogué, 2015, p. 141). Par la spatialité des interactions émotionnelles et sensibles entre les individus et les lieux, on envisage l'expérience polysensorielle du paysage dans un contexte géographique qui implique le visuel - et peut associer l'ouïe, le goût, l'odorat, le toucher - et va au-delà, en intégrant des multiples composantes esthétiques, intellectuelles et émotionnelles. Antoni Luna et Isabel Valverde (2015) appellent ainsi « paysages affectifs » des paysages dans lesquels des émotions servent de médiateurs aux nouvelles relations entre les individus et le monde. Comme indiqué par Joan Nogué (2015), le contenu immatériel dense des paysages est sans doute uniquement accessible via l'univers des émotions. Cette perspective est à rapprocher d'une tradition de paysagistes pour lesquels le paysage est avant tout une perception (recueillie par enquête afin d'identifier les valeurs et les images que les personnes ont sur le milieu ${ }^{6}$ ) qui peut aider à caractériser ce que sont les liens sensibles établis avec le paysage (Terrasson et Le Floch, 1995).

16 L'approche de la géographie émotionnelle, relativement récente, reste encore peu appliquée aux contextes lacustres ${ }^{7}$. Nous retiendrons cette approche sensible du paysage lacustre comme tout aussi pertinente que celle, plus classique, centrée sur les 
relations objectivées des sociétés à leur environnement ; la perspective est d'envisager leur possible articulation à partir de l'étude du lac d'Aiguebelette. Travailler à l'identification des interactions entre usages spatiaux objectivés du lac et expériences sensibles du paysage lacustre nécessite au préalable de se doter d'une représentation pouvant tenir lieu de cadre de référence géographique.

\section{Une cartographie des aires d'influence du paysage lacustre par le prisme du tourisme}

17 Le lac d'Aiguebelette est un lac de piedmont d'origine glaciaire ; il s'insère donc dans un espace situé au pied d'une montagne et entretient des rapports étroits avec celle-ci. C'est en se basant sur ce relief que l'on situe l'analyse du paysage, inscrivant le lac dans un cadre de verdure: délimité à l'Est par les monts boisés de la chaîne de l'Épine plongeant dans le lac en pentes abruptes, au Sud par le plateau de Lépin, au Nord par celui de Novalaise et, sur la rive Ouest, par les plages enherbées et la falaise de la Crusille. Dans ce cadre, on s'intéresse ensuite à la place des aménagements qui organisent les usages touristiques dans cet espace. La démarche retenue part d'une

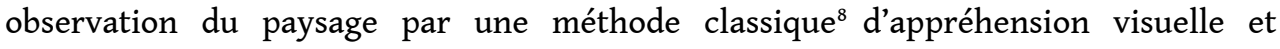
d'inventaire avec prise de photos sur le terrain. Le parcours de visites a été défini à partir des routes qui permettent d'approcher le lac, mais aussi, de manière plus distanciée, depuis les routes situées sur les versants, de façon à voir l'ensemble de l'espace lacustre afin d'identifier différentes zones de vue sur le lac. La description de la zone ainsi couverte est figurée sur une carte sur laquelle les éléments d'observation in situ sont positionnés en tant que composantes spatiales matérielles associées aux aménagements touristiques et ayant un impact visuel sur le paysage. Une deuxième démarche consiste à enrichir la représentation des éléments de topographie et du couvert végétal de façon à tenir compte des aires de vue sur le lac. Au final, à l'aide d'un système d'information géographique et de l'utilisation d'un modèle numérique de terrain, nous avons cartographié le paysage lacustre visible en fonction de l'altitude et du couvert végétal entourant le lac, ce qui permet de délimiter les zones d'approche et de vue sur le lac ainsi que la diversité des points de vue remarquables (cf. figure 2). On délimite ainsi une grande aire de vue sur le lac d'Aiguebelette de part et d'autre de celui-ci, avec une partie principalement cachée par la végétation sur sa partie Est, correspondant à l'emprise de l'espace protégé de la RNR. 
Figure 2. Zones de vue sur le paysage lacustre du lac d'Aiguebelette

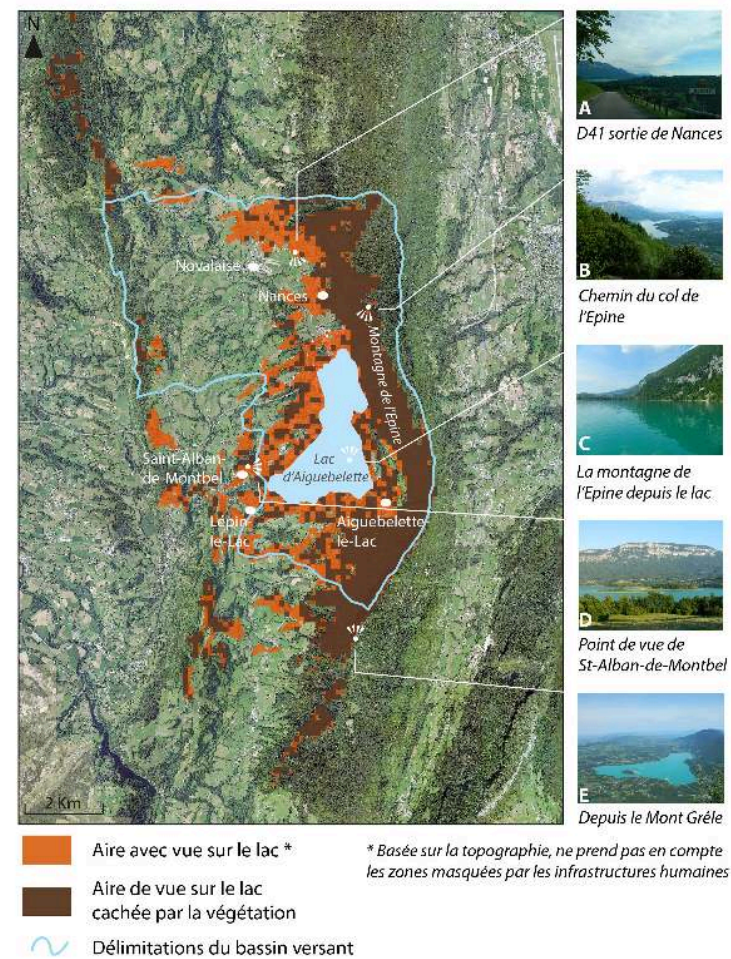

Sources : IGN (bases de données ORTHO, ALTI, TOPO) ; crédits photos et réalisation par les auteurs 2017

18 Sur cette base cartographique, différents espaces touristiques, dits aires d'influence $d u$ paysage lacustre, sont identifiés (cf. figure 3). En premier lieu, une aire d'influence lacustre in situ (dont on trouve une vue figure 2, C) correspondant à l'emprise spatiale du lac : il s'agit de la zone où le lac tient la place la plus importante dans le paysage, dans toute son étendue ou bien en partie en fonction de la morphologie du lac; depuis cette zone sont visibles, par exemple, les roselières et les îles du lac d'Aiguebelette qui participent à son image de lac naturel. 
Figure 3. Les aires d'influence du paysage lacustre à partir des aménagements touristiques

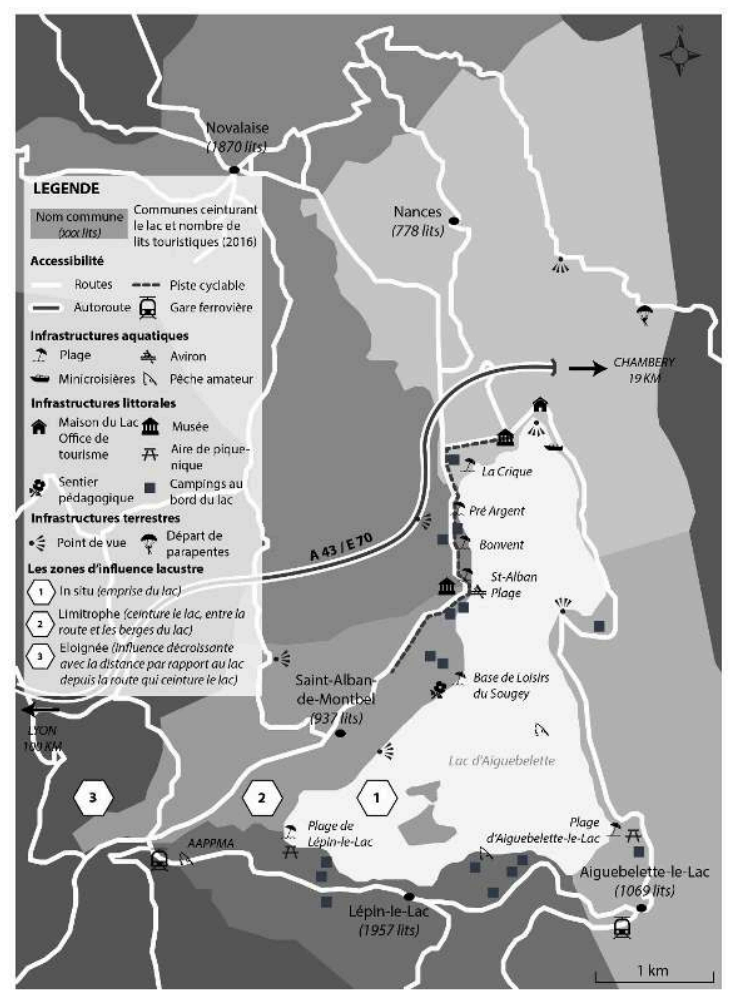

Sources : Observatoire Savoie Mont Blanc Tourisme, base de données APIDAE, Internet; observations et réalisation des auteurs, 2018

Ensuite, l'aire d'influence lacustre limitrophe qui ceinture le lac, située entre le lac et la route départementale permettant de faire le tour du lac d'Aiguebelette, qui correspond à la zone la plus aménagée, essentiellement dans sa partie Nord-Ouest, à proximité des accès routiers. Dans cet espace se trouve l'essentiel des équipements et aménagements nécessaires aux activités nautiques (plages, bases de loisirs), à la découverte du lac (maison du lac, musées) et aux prestataires de campings, restaurants et hôtels. La vue sur le paysage lacustre diffère en fonction de la rive du lac. Ainsi, le paysage lacustre depuis de la rive Ouest permet d'observer le lac et la montagne de l'Épine, très végétalisée, peu anthropisée ( $\mathrm{du}$ fait de la présence de la réserve, qui contraint l'emprise humaine) et culminant à $1427 \mathrm{~m}$ ( $c f$. figure 2, D). Le paysage lacustre visible depuis la rive Est se caractérise quant à lui par la présence de monts et de vallées à dominante agricole et faiblement urbanisés, seulement perceptibles en prenant de la hauteur sur la rive opposée ( $c f$. figure 2, B). La rive Nord permet d'apprécier des caractéristiques des deux points de vue précédents : une vue sur les massifs préalpins en arrière-plan et sur le lac dans son intégralité à certains endroits ; tout comme la rive Sud, qui permet également d'observer les deux îles du lac.

Enfin, une aire d'influence lacustre éloignée, plus ou moins étendue en fonction du relief, est caractérisée par un territoire où le lac occupe de moins en moins de place dans le paysage lacustre du fait de la distance à celui-ci (cf. figure 2, A), lac qui demeure cependant visible en grande partie ou dans son intégralité depuis les points les plus en altitude ( $c f$. figure 2, E). Cette zone présente quelques points de vue aménagés sur le lac, avec la présence de tables d'orientation, de tables de pique-nique ou de bancs au bord des routes et chemins, accessibles par des activités récréatives terrestres et la route. On 
y retrouve également les départs d'activités de loisirs terrestres (sentiers pédestres balisés) et aériens (aires de décollage de parapente) en lien avec le lac, ainsi que des hébergements touristiques; cette zone d'influence lacustre peut s'étendre très loin dans les zones de montagne.

21 Ces différentes aires d'influence du paysage lacustre, associées à des pratiques touristiques, sont des vecteurs de différentes représentations du lac et de son territoire.

\section{Les pratiques touristiques comme clés de spécification des paysages lacustres}

Les représentations cartographiques des zones de vue et aires d'influence du paysage lacustre révèlent la marque de l'empreinte touristique de ce territoire. Ce cadre paysager peut alors être mis en perspective avec les pratiques touristiques et récréatives identifiées sur le lac d'Aiguebelette. La méthodologie a consisté à collecter et analyser des données statistiques et documentaires pour identifier les caractéristiques générales de la destination Pays du lac d'Aiguebelette. Ces données de cadrage contextuel sur l'économie touristique ont été complétées par une analyse des pratiques touristiques telles qu'issues des relevés d'observations conduites in situ, portant sur la spatialisation des usages et des aménagements touristiques dans le cadre paysager étudié; l'objectif étant l'identification des pratiques qui se déploient sur et autour du lac en fonction des différentes aires d'influence du paysage lacustre.

Concernant les données de cadrage, dans la communication documentaire produite notamment par l'office du tourisme du Pays du lac d'Aiguebelette, la destination est promue, d'une façon générale, comme étant facilement accessible, à la fois en termes de transport, de prix et d'offres pour tous publics (Pays du lac d'Aiguebelette, 2017). À l'échelle du territoire, on compte environ 17300 lits touristiques, qui se partagent pour moitié entre lits non marchands et marchands, et au sujet desquels les professionnels soulignent la diversité de l'offre avec une relative montée en qualité, le développement des chambres d'hôtes et des réservations Airbnb (ibid.). Les différents usagers (habitants, résidents secondaires, touristes et excursionnistes) partagent (bon gré mal gré) l'espace. Les activités qui s'exercent directement sur le lac sont les plus prisées, surtout en été. Le lac est notamment connu pour la pratique de l'aviron: il dispose d'une base internationale qui permet d'accueillir des évènements de compétition, mais cette activité représente également une importante source de conflits autour des aménagements qu'elle suscite. C'est, plus largement, sur les possibilités de baignade, de détente et de pratiques de pleine nature que les professionnels de la destination PLA communiquent. Le lac accueille 10 plages, dont 7 payantes, qui enregistrent chaque année plus d'entrées ( 300000 pour les seuls trois mois de l'été $2018^{\circ}$ ) et connaissent des périodes de forte fréquentation se traduisant, certains week-ends d'été, par une saturation des plages et des parkings ${ }^{10}$. L'activité de pêche récréative est une autre pratique emblématique ${ }^{11}$ mais elle reste peu organisée et les droits de pêche sont complexes, ce qui génère des conflits d'usage. À ces activités s'ajoutent des activités économiques de location de pédalos, de paddles, de canoés et de barques qui complètent les revenus des entrées de plage. Cette économie, jointe à celle des hébergements (dont l'activité est évaluée en 2017 à 300000 nuitées marchandes), de la restauration (une quarantaine de restaurants) et des prestataires existant autour du lac 
et de façon diffuse sur le territoire du PLA, totalise un chiffre d'affaires annuel direct de $20 \mathrm{M} €$ et un volume de 200 emplois directs estimés pour l'ensemble de la destination ; chiffres auxquels il faudrait ajouter les emplois et revenus indirects, et notamment la valorisation d'une économie de terroir (producteurs de vins de Savoie, de fromages) avec trois magasins de coopératives et tout un ensemble de productions locales (miel, noix, charcuteries, fromages de chèvre, safran, escargots, gâteaux et glaces).

Concernant les pratiques identifiées, elles s'organisent selon des règles de spatialisation qu'il est possible d'analyser à partir de la typologie proposée des zones d'influence du paysage lacustre (cf. figure 3 ) :

- Dans l'aire d'influence lacustre in situ (cf. figure 3, 1), les pratiques sont spatialisées de façon différente entre: le milieu littoral, qui concerne davantage la baignade, le pédalo voire le paddle ; le milieu pélagique (partie lacustre de pleine eau, éloignée des rives et du fond du lac) destiné à la pêche récréative, l'aviron, la natation pour la traversée du lac, le canoé, le kayak, la voile; et le milieu benthique (à l'interface entre l'eau et le substrat) réservé à la plongée. Pour ces pratiques, un accès physique et visuel au lac est nécessairement requis. Les propriétés physiques (p.ex: la houle, la température de l'eau), biologiques (p.ex: la présence de poissons) ou écologiques (p.ex: la qualité de l'eau, la présence d'habitats spécifiques) du lac sont recherchées.

- L'aire d'influence lacustre limitrophe ( $c f$. figure 3, 2) est investie par les pratiques balnéaires et de camping ainsi que par les nombreux événements festifs, culturels et sportifs autour du lac. Le contact avec le lac peut être physique et/ou visuel, en fonction de la pratique et de la vue sur la rive du lac où l'usager se trouve. Le contact peut également être très limité, voire inexistant, dans le cadre d'activités d'intérieur (p. ex. les musées). Cette zone d'influence concerne essentiellement les activités associées aux plages et bases de loisirs (pique-nique, aire de jeux pour enfants, trampoline, mini-golf, accrobranche, contemplation, lecture, bronzage, etc.), les activités ou championnats sportifs organisés autour du lac (marche, vélo, jogging, marche afghane, course à pied, etc.), les activités en lien avec les aires de campings et les autres hébergements et équipements de restauration, les évènements festifs ou encore les activités d'interprétation environnementale et de découverte patrimoniale qui mobilisent un contenu éducatif (balade thématique, visite guidée, sortie nature, visite de la maquette de la cité lacustre du Néolithique ou le parcours spectacle à la Maison du lac, les expositions au Musée lac et nature). Ces activités éducatives proposent une diffusion des connaissances et une sensibilisation à l'importance écologique et biologique des milieux naturels ; elles valorisent le patrimoine local et la présence des sites palafittiques auprès des habitants et des touristes.

- Enfin, au sein de l'aire d'influence lacustre éloignée (cf. figure 3,3), se déploient les activités de pleine nature sur les sentiers (randonnée pédestre, équestre, VTT, parapente), les activités de tourisme rural, de marchés et d'animations culturelles dans les petits villages; on y recense également des hébergements touristiques plus éloignés du lac (gites et chambres d'hôtes). Le contact physique avec le lac est absent et le contact visuel importe de moins en moins; il devient sporadique avec la distance et s'appuie sur la présence de points de vue depuis le Mont Grêle, le chemin du col de l'Épine et la route D41 (cf. figures 2, A, B, E). Un contact que l'on pourrait qualifier de « cognitif » est néanmoins très présent et participe à la perception du paysage lacustre et du territoire.

L'ensemble de ces pratiques touristiques et récréatives révèle une continuité spatiale entre les différentes aires d'influence où le lac est mobilisé en tant qu'objet physique appréhendé de façon visuelle dans une posture contemplative, mais aussi 
polysensorielle et cognitive dans le cadre des activités en contact avec l'eau et/ou avec son cadre environnemental. Il reste à identifier auprès des usagers et des acteurs de l'espace lacustre de quelle façon ces multiples dimensions participent aussi d'une explicitation du paysage du lac d'Aiguebelette.

\section{L'expérience polysensorielle du paysage lacustre par les acteurs et usagers du territoire}

26 L'approche de la géographie émotionnelle reconnaît le rôle des sensations pour exprimer le rapport à l'espace vécu, qui sont révélées à travers des récits d'expérience du paysage. Leur analyse est traduite en représentations; pour cela, on utilise une méthodologie d'entretiens épisodiques avec des individus retenus en tant qu'usagers ou acteurs des lieux (Flick, 2009). L'entretien permet d'encourager la narration de faits ou d'épisodes significatifs choisis par les individus interrogés et d'accéder à la connaissance narrative-épisodique et sémantique-conceptuelle contenue dans le récit de l'expérience. Dans cette perspective, une trentaine d'entretiens ont été conduits auprès d'acteurs et d'usagers du lac d'Aiguebelette : acteurs institutionnels du tourisme (office du tourisme, Agence touristique départementale de la Savoie, Savoie Mont Blanc Tourisme, Auvergne Rhône Alpes Tourisme), acteurs publics intervenant à différentes échelles territoriales (Syndicat mixte de l'Avant-pays savoyard, gestionnaires, techniciens et élus de la CCLA), Chambre d'agriculture Savoie Mont Blanc, Université Savoie Mont Blanc, propriétaires du lac (famille Rivérieulx de Chambost de Lépin et Électricité de France [EDF]), prestataires privés du tourisme (hébergeurs, restaurateurs, opérateurs multi-activités), acteurs associatifs (associations écologiques, sportives, culturelles), touristes et habitants. Ces entretiens ont été réalisés à partir d'un guide de questions ouvertes, soit à l'occasion de rendez-vous (avec les acteurs institutionnels, publics, privés, associatifs, autres), soit à partir de rencontres in situ faites au hasard (avec les touristes et les habitants). L'enregistrement sonore et la transcription des contenus des entretiens ont permis un traitement des données d'enquête par codage thématique (Flick, 2009) en utilisant le logiciel Sonal 2.0.77. L'analyse des données établit des proximités de perception et d'expériences entre individus, que nous avons organisées en trois axes de représentations dominantes associées au paysage lacustre. Précisons que ces représentations ne sont pas des catégories clairement différenciées ; il ne s'agit donc pas d'une typologie, mais d'une analyse par tendances sémantiques qui ne s'opposent pas entre elles et peuvent présenter des éléments partagés ponctuellement par des acteurs ou usagers.

On propose ainsi une synthèse des représentations du paysage lacustre d'Aiguebelette à partir de trois axes principaux de représentations : un premier axe qui s'établit en référence au lac naturel, un second axe centré sur le lac comme lieu de contrastes d'expériences et, enfin, un axe consacré au lac comme objet de contraintes et de conflits.

\section{Le lac comme expérience du « cadre naturel »}

La qualité de lac naturel fonde l'essentiel des perceptions et les acteurs et usagers rapportent l'importance pour eux de sa préservation. Le lac d'Aiguebelette se distingue par des éléments physico-sensoriels présentés comme spécifiques : l'absence de vent, la température et la couleur vert-turquoise de l'eau, la diversité des formes et textures 
présentes dans la composition du cadre végétal et forestier environnant le lac, enfin, la présence des montagnes. Tous ces éléments sont cités de manière récurrente comme étant porteurs d'expériences polysensorielles du paysage lacustre et sont rapportés à partir de pratiques présentées comme singulières et qualitatives, possibles là mieux qu'ailleurs. Ce sens du caractère particulier, voire exceptionnel, du cadre est associé, dans les perceptions, à une idée globale d'esthétique du paysage, décrit comme un " écrin ", un décor visuellement attrayant par la composition formée par le lac dans un ensemble plus large associant la végétation, par le contraste ou l'harmonie des couleurs, des reliefs et des ambiances. Potentiellement, toutes les aires d'influence du paysage lacustre sont ici concernées, le lac étant considéré comme un ensemble paysager «naturel ». Mais, comme l'a explicité Augustin Berque (2009), cette beauté du paysage n'est pas dans la nature elle-même ; elle est le produit de l'appréciation issue de l'expérience de l'observateur. La description proposée se réfère donc implicitement aux valeurs sociales et symboliques liées aux modèles et normes esthétiques construits de longue date dans le regard porté sur les lacs alpins, ainsi qu'à l'ambiance du cadre montagnard en arrière-plan (Liabeuf, 2009 ; Vernex, 2009).

C'est tellement beau, on n'a pas de mots, les gens qui viennent ici, sont admiratifs (...). C'est pour ça, il faut le garder tel qu'il est, il n'y a pas de mots, tellement c'est beau. Vous allez d'un côté, vous voyez le lac, il n'y a pas que le lac, et il y a la montagne, c'est magnifique... On en a plein les yeux (...). (un élu local).

Ce qu'on a trouvé très beau, c'est la couleur du lac, la couleur extraordinaire; et puis le retour le long du lac, il est très bien ombragé (par le versant...). (un touriste).

Les récits révèlent des expériences à partir de différents points de vue sur le paysage :

(...) on a une vue aérienne, c'est-à-dire qu'il y a le paysage quand on est au bord de l'eau et il y a le paysage quand on est en altitude (...). Ça veut dire qu'il y a une variété de paysages, parce qu'il y a autant de paysages qu'il y a de points de vue, il y a beaucoup de points de vue parce qu'on peut être à différentes altitudes, donc ça c'est une particularité (...). (un acteur institutionnel du tourisme)

Les émotions et la densité sensible contenue dans les récits sous-tendent une expérience du paysage qui va au-delà de la perception visuelle :

Le lac, moi, je le vis tous les jours, et tous les jours je le trouve différent, et tous les jours je le trouve magnifique. Il y a toutes les saisons (...), quand il y a la neige en hiver et qu'on a une journée ensoleillée, on sort avec les bateaux et on rame à 2-3 degrés mais, comme on fait du sport, on n'a pas froid et on est dans cet environnement où on a à la fois le soleil, le lac et la neige tout autour. C'est vraiment magnifique. C'est le côté quand il fait beau. Et puis, on a aussi ces journées où il y a du brouillard (...). Moi, il m'est arrivé, à titre personnel, de prendre ma barque et d'aller jusque sur l'île là-bas et de regarder la brume du matin qui commence à se lever sur le lac, c'est magique! Il y a plein de situations, ça change tout le temps. (un acteur d'une association sportive)

\section{Le lac comme lieu de contraste d'expériences}

31 L'une des particularités du lac d'Aiguebelette, par rapport à l'ensemble des lacs alpins, est qu'il se présente comme un "lac de campagne »: ici, pas de ville au bord de l'eau, seulement un maillage de petits villages qui restent peu visibles dans le paysage lacustre. En lien avec cet environnement peu urbanisé, les acteurs et usagers se réfèrent à une ambiance de retrait, de confins, décrite par des termes comme: " calme », « tranquillité ", « sérénité », « quiétude ", « ressourcement », " apaisement », 
« repos ", ou encore : "inspiration à la créativité », « bien-être », « contemplation », " confidentialité », « intimité dans un site caché », " mystérieux ».

Devant le lac, on éprouve quelque chose qui est assez intime, c'est-à-dire qu'un peu comme Lamartine l'a fait au lac du Bourget, on est dans la contemplation (...). Ça génère beaucoup de choses à l'intérieur, parce que cette beauté qui rentre par les yeux, finalement, elle nous pénètre et elle nous apporte du calme et de la sérénité (...). (un prestataire privé du tourisme)

Ces propos d'acteurs et d'usagers décrivant leurs rapports au lac sont associés à des sentiments d'appartenance, de privilège d'avoir accès à des lieux remarquables pour leur « qualité de vie », une « carte postale vivante ", un lac « à taille humaine ».

Mais ces expressions d'harmonie, qui prévalent s'agissant du paysage considéré en tant qu'entité, sont contrebalancées par des récits faisant surgir des expériences plus diverses au sein des différentes aires d'influence ainsi que par les difficultés à tenir compte des expériences relevant du lac naturel avec celles du lac touristique. Dans les aires d'influence in situ et limitrophes, les difficultés se cristallisent autour de la nonconcordance des usages. Les abords du lac sont valorisés pour satisfaire le désir de proximité de l'eau pour les touristes. Mais, la présence de la RNR et la gestion privée des plages l'été, délimitent fortement les modalités d'accès au lac. Par ailleurs, l'espace est investi à la fois par des sportifs, des touristes, des excursionnistes, des résidents secondaires, des habitants, des collectifs environnementaux, des pêcheurs récréatifs, etc., dont la diversité des pratiques suppose de combiner les différents intérêts et nécessite de trouver des compromis, des solutions de partage des espaces, et nécessite aussi de poser des limites en réponse aux pressions exercées sur le milieu par une fréquentation de masse en saison estivale.

Donc ça, c'est très difficile à gérer, parce qu'il y a beaucoup, beaucoup de monde sur une seule journée et du coup ça transforme complètement le lac... On se dit que là, ce n'est plus « notre lac ", c'est déjà autre chose, c'est une énorme piscine, il y a des voitures de partout, il y a du bruit, voilà ! (un prestataire privé du tourisme)

\section{Le lac comme objet de contraintes et de conflits}

L'expérience sensible du paysage du lac d'Aiguebelette est faite de ces articulations complexes entre espaces et pratiques, entre temps du quotidien et des saisons, entre vécu solitaire et trop-plein d'usages. Les acteurs expriment un sentiment de contrainte et la difficulté à faire avec cette complexité. L'expérience du cadre se réfère alors aux modalités de préservation et de gestion du lac, qui sont évoquées de façon récurrente par les acteurs. Les propriétaires, gestionnaires et autres acteurs locaux (office du tourisme, prestataires privés du tourisme et associations) témoignent assez généralement de l'enjeu de maintenir l'état de préservation du lac pour conserver la qualité de ses eaux et des milieux naturels. Ils font référence à la réglementation qui encadre les usages et activités (p. ex, l'usage des embarcations à moteur thermique est interdit sur ce plan d'eau ${ }^{12}$ ). Ils connaissent aussi l'existence de dispositifs de protection qui se sont «imposés " par étapes. La création de la RNR sur l'ensemble du lac et la montagne de l'Épine voisine située dans son bassin versant a conforté la réglementation et la gouvernance locale en faveur de la protection de la biodiversité et contribué à préserver la qualité des eaux du lac, de ses milieux aquatiques et la richesse de ses zones humides. L'objectif de ce classement est de labelliser le site en cohérence avec la logique écotouristique ${ }^{13}$; sa gestion admet certains usages sous condition de respect du site et des équilibres environnementaux. La CCLA et le Conservatoire des 
espaces naturels de Savoie (CENS) assurent conjointement sa gestion. Cette expérience du lac géré est souvent commentée comme une contrainte jugée nécessaire mais néanmoins sujette à critique. La crainte de «sanctuarisation du lac » est évoquée par les acteurs rencontrés et l'existence de la RNR est toujours confrontée au défi de son appropriation par les habitants, les acteurs et le public des usagers du lac en général. En période de fréquentation touristique, les incivilités, les réactions au contrôle et les confrontations avec les gardes de la RNR sont récurrentes:

Donc, c'est très conflictuel! C'est un lieu calme au niveau du paysage mais au niveau des rapports humains, des relations humaines, assez contradictoirement, ça peut générer beaucoup de conflits. (un ancien élu local)

Dans l'aire d'influence limitrophe et l'aire d'influence in situ, les sources de conflits d'usages et de partage de l'espace sont nombreuses; elles se rapportent notamment à l'opposition entre la pratique de l'aviron et de la pêche récréative et impliquent les associations de protection et de conservation de la nature; elles concernent également les problèmes de compatibilité de fonctions de la ressource en eau du lac entre la production d'hydroélectricité et les pratiques de la pêche et du tourisme.

Dans ce contexte, les perceptions sociales associées au paysage lacustre, portées notamment par les élus et acteurs économiques, évoluent : les collectivités ont engagé une stratégie qui tente de combiner les intérêts en termes de protection de la nature et de développement touristique. Dans un territoire où le tourisme est pensé globalement comme une opportunité de développement durable, le lac est potentiellement une entité géotouristique fédératrice, au sein de laquelle le paysage et sa structuration en aires d'influence pourraient servir de support de concertation.

\section{Conclusion}

En proposant une caractérisation des aires d'influence du paysage lacustre, nous avons associé celui-ci à l'analyse de la spatialisation des pratiques touristiques et de loisirs et à la prise en compte des axes de représentation porteurs de sensibilité et d'enjeux sociétaux. Cette proposition a été construite dans une conception qui articule les dimensions sociétales et environnementales du paysage lacustre. Elle s'inspire de l'approche géographique qui vise à concilier l'existence physique du lac, en tant que géo et écosystème, avec sa réalité sociale en tant qu'espace d'usages et de vécu, mais également avec les perceptions sensibles des individus qui le pratiquent. Cette approche, qui emprunte à des méthodologies assez différentes, est inspirée des travaux d'Augustin Berque et de ceux conduits sur l'observation des lacs alpins par Jean-Claude Vernex, à qui l'on doit l'analyse de la place des lacs au sein des écosystèmes humains. À partir de ces travaux, notre proposition a combiné l'analyse spatiale des pratiques touristiques et de loisirs et celle de l'expérience individuelle des lieux et a ainsi permis d'articuler des faits objectivés (à travers la cartographie et la spatialisation des pratiques) et des contenus subjectifs traduits en représentations.

La combinaison de ces différentes dimensions du paysage lacustre révèle des difficultés : alors que les lacs sont de plus en plus promus en tant qu'espaces investis par la mise en tourisme, le cas du lac d'Aiguebelette montre la complexité de faire cohabiter des pratiques et des expériences dans un espace où la protection des milieux est une donnée importante. Cela entraîne des conflits qui ne sont pas simples à résoudre et qui nécessitent de poser les bases minimales d'un espace commun à la fois 
naturel et social. La dimension "naturelle» prend en compte les composantes physiques du milieu et leur fonctionnement écosystémique, ainsi que les modalités d'impact et de réponse aux pressions liées à la fréquentation; la dimension "sociale " permet d'aborder les usages et l'évolution du cadre paysager et de s'intéresser également aux expériences sensibles, notamment esthétiques. Des interactions constantes ont lieu entre ces approches; un problème méthodologique persistant est celui de leur articulation simultanée dans une démarche d'observation combinant le fonctionnement du milieu, les pratiques, les représentations, ce à quoi il conviendrait d'ajouter le jeu des acteurs, l'identification de leurs intérêts spécifiques et de leurs projets sur l'espace et les modalités de la gouvernance d'ensemble.

\section{BIBLIOGRAPHIE}

James ARONSON et Édouard LE FLOC'H, « Que faire de tant de notions de paysage ? ", Natures Sciences et Sociétés, vol. 4, nº 3, 1996.

Robert BERGERON, « Le Lac des Sapins, un aménagement pour les loisirs et le tourisme en HautBeaujolais ", Hommes et Terres du Nord, n 4 : Tourisme et développement local et régional, 1987. Robert BERGERON et Marie-Andrée BUISSON, « Les plans d'eau récréatifs en moyenne montagne : l'exemple de Rhône-Alpes », Revue de géographie de Lyon, vol. 63, nº 4, 1988.

Augustin BERQUE, El pensamiento paisajero, Editorial Biblioteca Nueva, 2009.

Augustin BERQUE, Les raisons du paysage de la Chine antique aux environnements de synthèse, Hazan, 1995.

Augustin BERQUE, « Paysage-empreinte, paysage-matrice : éléments de problématique pour une géographie culturelle », Espace géographique, tome 13, n 1, 1984.

Georges BERTRAND, «Le paysage entre la Nature et la Société », Revue géographique des Pyrénées et du Sud-Ouest, tome 49, n², 1978.

Jean-Claude BONTRON, Yves LUGINBÜHL et Zsuzsa CROS, Méthodologie pour l'identification et la typologie des paysages, ministère de l'Équipement (DAU)/SEGESA-STRATES/CNRS, 130, 1991.

Nathalie CAYLA, « Les processus de construction du géotourisme alpin », Revue Téoros, vol. 29, $\mathrm{n}^{\circ} 2$, 2010.

Communauté de communes du lac d'Aiguebelette (CCLA), Pour un développement éco-touristique du territoire du lac d'Aiguebelette, 2009 (non publié).

Paul CLAVAL, La géographie culturelle, une nouvelle approche des sociétés et des milieux, coll. «U. », Armand Colin, 2012.

Paul CLAVAL, «Champ et perspectives de la géographie culturelle », Géographie et cultures, vol. 1, $\mathrm{n}^{\circ} 1,1992$.

Denis COSGROVE, « A terrain of metaphor: cultural geography, 1986-1989 », Progress in Human Geography, vol. 13, n 4, 1989. 
Mélanie DUVAL et Christophe GAUCHON, « Tourisme, géosciences et enjeux de territoires : actualités du géotourisme ", Revue Téoros, vol. 29, n², 2010.

Claudio FERRATA, « La fabrication du paysage dans la région des lacs du sud des Alpes », Le Globe. Revue genevoise de géographie, vol. 147, 2007.

Uwe FLICK, Introdução à pesquisa qualitativa, Artmed, 2009 (3édition).

Michel JARGOT et Jean LOUP, « Un cas particulier de pollution lacustre : le lac d'Aiguebelette », Revue de géographie alpine, tome 71, n 4, 1983.

Owain JONES, « An ecology of emotion, memory, self and landscape », dans Joyce DAVIDSON, Liz BONDI et Mick SMITH (dir.) Emotional Geographies, Routledge, 2016.

Jean-Pierre LOZATO-GIOTART, Géographie du tourisme. De l'espace consommé à l'espace maitrisé, Pearson Education France, 2008.

Bertrand LÉvY, Rafaël MATOS et Sven RAFFESTIN, «L'évolution de la représentation du Léman à travers les guides et la promotion touristiques du XIX siècle à nos jours : le cas genevois ", Le Globe. Revue genevoise de géographie, tome 138 : Le lac, regards croisés, 1998.

Brigitte LIABEUf, «Avec vue sur lac », dans Brigitte LIABEUf, Sophie MARIN et Yann BAZIN (dir.), Avec vue sur lac : regards sur les lacs alpins du XVIII siècle à nos jours, Fage Éditions, Musée-Chateau, 2009.

Yves LUGINBÜHL, « Paysage élitaire et paysages ordinaires », Ethnologie française, tome XIX, $\mathrm{n}^{\circ} 3$, 1989.

Antoni LUNA et Isabel VALVERDE, « Presentación. Afecto, sentido, sensibilidad: miradas transversales sobre paisaje y emoción », dans Antoni LUNA et Isabel VALVERDE (dir.), Teoría y paisaje II: Paisaje y emoción. El resurgir de las geografías emocionales, Observatorio del Paisaje de Cataluña et Universitat Pompeu Fabra, 2015.

Joan NOGUÉ, « Emoción, lugar y paisaje », dans Antoni LUNA et Isabel VALVERDE (dir.), Teoría y paisaje II: Paisaje y emoción. El resurgir de las geografías emocionales, Observatorio del Paisaje de Cataluña et Universitat Pompeu Fabra, 2015.

Pays du lac d'Aiguebelette, Stratégie de développement touristique :état des lieux, diagnostic, éléments prospectifs, présentation en réunion plénière, 30 mai 2017 (non publié).

Henri ROUGIER, « Essai de typologie des lacs alpins », Bulletin de l'association de géographes français, vol. 4, décembre : Géographie des lacs, 2000.

Gabriel ROUGERIE et Nicolas BEROUTCHACHVILI, Géosystèmes et paysages. Bilan et méthodes, Armand Colin, 1991.

Daniel TERRASSON et Sophie LE FLOCH, «La notion de paysage : au-delà des formes, les liens entre les hommes et les territoires ", Natures, Sciences, Sociétés, n 3, 1995.

Michel TissuT, « Les différentes facettes du concept de patrimoine : compatibilité avec des projets de développement durable », Bulletin de l'Association de géographes français, vol. 3, septembre :

Terroirs, patrimoine et pays, 2002.

Michel TIssut, « Les perspectives de développement durable dans le bassin du lac d'Aiguebelette (Savoie) ", Bulletin de l'association de géographes français, vol. 4, décembre : Géographie des lacs, 2000.

Laurent tOUCHART, "Qu'est-ce qu'un lac ? ", Bulletin de l'Association de géographes français, vol. 4, décembre : Géographie des lacs, 2000. 
Laurent TOUCHART, «La machine lacustre : l'exemple du Léman », Annales de géographie, tome 102, $n^{\circ}$ 573, 1993.

Jean-Claude VERNEX, « Le beau paysage lacustre : archéologie d'un regard » dans Brigitte LIABEUF, Sophie MARIN et Yann BAZIN (dir.), Avec vue sur lac : regards sur les lacs alpins du XVIII siècle à nos jours, Fage Éditions, Musée-Château, 2009.

Jean-Claude VERNEX, « Qu'est-ce qu'un lac ? De l'imaginaire lacustre à l'aménagement ». Le Globe. Revue genevoise de géographie, vol. 138, n 1, 1998.

Jean-Claude VERNEX, «L'invention des lieux touristiques ». Le Globe. Revue genevoise de géographie, vol. 133, n 1, 1993.

Jean-Claude VERNEX « Évolution des pratiques de loisirs et espace littoral lacustre. Une étude de cas : le lac d'Annecy (France) », Le Globe. Revue genevoise de géographie, vol. 129, n 1, 1989.

Jean-Claude VERNEX, «Paysage et société : l'exemple du littoral du Lac d'Annecy », Le Globe. Revue genevoise de géographie, vol. 125 : Les Alpes dans le temps et dans l'espace, 1985.

Jean-Claude VERNEX et Michèle MARTIN, «Vendre le lac : quelles images, quels imaginaires? L'exemple d'Annecy », dans Brigitte LIABEUF, Sophie MARIN et Yann BAZIN (dir.), Avec vue sur lac : regards sur les lacs alpins du XVIII siècle à nos jours, Fage Éditions, Musée-Chateau, 2009.

\section{NOTES}

1. En fait dès 1884, un tronçon est ouvert par la compagnie ferroviaire de Paris-LyonMéditerranée relie Chambéry à Saint-André-le-Gua (devenu Saint-André-le-Gaz) en Isère et permet de desservir le lac d'Aiguebelette.

2. Le lac est un lac privé qui appartient aujourd'hui à la famille de Rivérieulx de Chambost de Lépin et à Électricité de France (EDF), qui ont confié sa gestion à la Communauté de communes du lac d'Aiguebelette (CCLA).

3. La CCLA regroupe les communes suivantes : Aiguebelette-le-Lac, Attignat-Oncin, Ayn, Dullin, Gerbaix, Lépin-le-Lac, Marcieux, Nances, Novalaise et St-Alban de Montbel ; elle est en charge, dans le cadre de sa compétence «actions de protection et de mise en valeur de l'environnement", de la gestion du lac d'Aiguebelette et de ses usages (notamment: réglementation des ports et de la navigation, pratique de pêche et aviron, occupation des berges, plongée et baignade) et, dans le cadre de sa compétence «actions de développement économique ", de l'aménagement, l'entretien et la gestion des zones de loisirs (base du Sougey, base d'Aiguebelette-le-lac, ports sur Aiguebelette-le-Lac et Nances) (source: http:// www.ccla.fr/).

4. La science des lacs, née à la fin du XIX ${ }^{\mathrm{e}}$ siècle, définit le lac comme « une masse d'eau stagnante sans communication directe avec la mer, située dans une dépression du sol fermée de tous côtés » (Forel, 1892, cité par Touchart, 2000).

5. Le géotourisme a été défini, dans une acception large, par la National Geographic Society comme un tourisme qui préserve et valorise le caractère géographique d'un lieu: son environnement, son patrimoine, sa beauté, sa culture et le bien-être de ses résidents.

6. Notons que la prise en compte des perceptions par les populations est aujourd'hui un enjeu inscrit dans la définition du paysage de la Convention européenne du paysage, signée à Florence le 20 octobre 2000, qui énonce que «le paysage désigne une partie de territoire telle que perçue par les populations, dont le caractère résulte de facteurs naturels et/ou humains et de leurs interrelations » (p. 2). 
7. On relève cependant le travail de Marion Ernwein et Anne Sgard (2012) qui se sont intéressées au paysage polysensoriel du Léman dans ses dimensions perceptives, émotionnelles et corporelles dans une étude visant à comprendre, avec les non-voyants, ce que peut être le « sentiment paysager» sans la vue.

8. Méthode généralement employée dans l'analyse des paysages (Bontron, Luginbühl et Cros, 1991).

9. Soit une hausse de $21 \%$ par rapport à l'année 2017.

10. On a recensé plus de 17000 personnes sur les plages le dernier week-end de juin 2019, au cours duquel les températures ont été caniculaires ; plages et parkings aux abords des lacs se sont trouvés saturés et des véhicules ont été refoulés (source : Le Dauphiné libéré, 30 juin 2019).

11. À l'échelle du PLA, ce sont 1500 cartes touristiques annuelles de pêche qui sont vendues (pour une pratique à la journée, en semaine ou découverte) (source : PLA, 2017).

12. En 1967, un arrêté préfectoral interdit la circulation des bateaux à moteur thermique et limite les vitesses de circulation (CCLA, 2009).

13. La CCLA s'est engagée en 2009 dans une démarche régionale de «territoire écotouristique exemplaire ».

\section{RÉSUMÉS}

L'espace lacustre, où interagissent des pratiques de loisirs et un environnement physique qui leur sert de support, permet d'éclairer le rôle du tourisme dans la configuration du paysage. À partir $\mathrm{du}$ cas du lac d'Aiguebelette (Alpes françaises), cet article propose une analyse géographique du paysage lacustre qui articule ses dimensions physiques et environnementales avec les pratiques et représentations associées à l'expérience des lieux par le prisme du tourisme. Trois résultats ressortent de cette étude : une cartographie des aménagements touristiques à partir d'un zonage du paysage d'influence lacustre; une analyse spatiale des pratiques touristiques en fonction de la proximité au lac; enfin, à partir de l'expérience sensible des acteurs et usagers du territoire, on caractérise les axes de représentations et enjeux portés sur le paysage lacustre. Cette approche permet finalement d'identifier la diversité des espaces, des pratiques touristiques et des expériences en lien avec l'environnement lacustre, élargissant ainsi la perspective d'analyse du paysage lacustre, en articulant ses dimensions sociétales et environnementales.

The lake area, where leisure practices interact with the physical environment that supports them, allows to clarify the role of tourism in the landscape configuration. From the case of Lac d'Aiguebelette (French Alps), this article proposes a geographic analysis of the lake landscape that articulates its physical and environmental dimensions with the practices and representations associated with the experience of places through the prism of tourism. Three results emerge from this study: first, we established a zoning of the landscape influenced by the lake based on a cartography of tourism infrastructures; a spatial analysis of tourism practices is then conducted according to the proximity to the lake; finally, interested in the sensitive experience of the lake's actors and users, we characterize the axes of representations and issues related to the lake landscape. This approach makes it possible to identify the diversity of tourism spaces, practices and experiences related to the lake environment, thus broadening the perspective of analysis of the lake landscape by articulating its societal and environmental dimensions. 
INDEX

Keywords : lake landscape, lake tourism, tourist practices, cultural geography, alpine lakes Mots-clés : paysage lacustre, tourisme lacustre, pratiques touristiques, géographie culturelle, lacs alpins

\section{AUTEURS}

\section{LAURA RUDZEWICZ}

Docteure en géographie, Université Federal do Rio Grande do Sul, UFRGS ; enseignantechercheure, Université Federal de Pelotas, UFPEL

Diplômée en tourisme

laurar.turismo@gmail.com

\section{YOANN BAULAZ}

Docteur en géographie, Université Savoie Mont-Blanc, CNRS, INRA, UMR EDYTEM, UMR CARRTEL Géographe et écologue

yoann.baulaz@gmail.com

\section{VÉRONIQUE PEYRACHE-GADEAU}

Maître de conférences HDR en géographie et aménagement, Université Grenoble Alpes, Université Savoie Mont Blanc, CNRS, EDYTEM

Socioéconomiste

veronique.peyrache-gadeau@univ-smb.fr 